\title{
Statistical Simulation of Low-Speed Rarefied Gas Flows
}

\author{
Jing Fan and Ching Shen \\ Laboratory of High Temperature Gas Dynamics, Institute of Mechanics, CAS, Beijing 100080, China \\ E-mail: jfan@imech.ac.cn, cshen@imech.ac.cn
}

Received June 12, 2000; revised November 15, 2000

Molecular-based numerical schemes, such as the direct simulation Monte Carlo (DSMC) method, are more physically appropriate for rarefied gas flows in microelectromechanical systems (MEMS). It is difficult for them to be statistically convergent, however, because the statistical fluctuation becomes insurmountably large at the low Mach numbers that are characteristic of MEMS. An information preservation (IP) technique is proposed to address this issue. This technique assigns each simulated molecule in the DSMC method two velocities. One is the molecular velocity used to compute the molecular motion following the same steps as the DSMC method. The other is called information velocity. It corresponds to the collective velocity of an enormous number of real molecules that the simulated molecule represents. Using the information velocity to compute macroscopic velocity and shear stress may remove the statistical fluctuation source inherent in the DSMC method that results from the randomness of the thermal velocity. The IP technique has been applied to benchmark problems, namely Couette, Poiseuille, and Rayleigh flows, in the entire Knudsen regime. The characteristic velocities in these flows range from 0.01 to $1 \mathrm{~m} / \mathrm{s}$, much smaller than the thermal velocity of about $340 \mathrm{~m} / \mathrm{s}$ at room temperature. The meaningful results are obtained at a sample size of $10^{3}-10^{4}$, in comparison with a sample size of $10^{8}$ or more required for the DSMC method at such a range of flow velocity. This results in a tremendous gain in CPU time. The velocity distributions, surface shear stress, and mass flux given by the IP calculations compare quite well with exact solutions at the continuum and free molecular limits, and with the numerical solutions of the linearized Boltzmann equation and experimental data in the transition regime. (C) 2001 Academic Press 


\section{INTRODUCTION}

Advances in micromachining technology have allowed successful fabrication of many microelectromechanical systems (MEMS). The characteristic size of MEMS ranges from submillimeter to submicrometer. This provides a tool with wide applications to observe, measure, and control a new world [1]. In the small-scale world, noncontinuum, rarefied gas flow phenomena become prevalent, which may make MEMS behave quite differently from their counterparts in macroscopic machinery.

To understand this kind of flow, basic experiments have been carried out, for example, those on microchannel gas flows [2-10]. The typical channel dimensions were about one micrometer high by several tens of micrometers wide by several thousands of micrometers long. The flow was driven by the pressure difference between the inlet and the outlet, with a velocity of about $0.2 \mathrm{~m} / \mathrm{s}$. The measured mass flux was higher than the Navier-Stokes (N-S) solution based on nonslip boundary condition [2-10]. When the slip velocity

$$
u_{s}=\left.\frac{2-\sigma}{\sigma} \lambda \frac{d u}{d n}\right|_{s}
$$

was introduced along the microchannel surfaces, and the tangential momentum accommodation coefficient $\sigma$ was properly chosen, the $\mathrm{N}-\mathrm{S}$ solution became in agreement with experiment $[8,9]$, where $\lambda$ is the molecular mean free path, and $\left.\frac{d u}{d n}\right|_{s}$ denotes the normal velocity gradient at the surfaces. As the noncontinuum effect increases further, kinetic theory indicates that the $\mathrm{N}-\mathrm{S}$ equations become invalid. This is also verified by microchannel experiment [9]: A significant discrepancy between the slope of the measured and slip Navier-Stokes flow conductances is found as the outlet pressure of helium flows decreases.

Molecular-based schemes are more physically appropriate for rarefied gas flows. The most popular approach is the direct simulation Monte Carlo (DSMC) technique that was developed and popularized in the context of hypersonic aerospace applications [11]. Some researchers have applied it to microchannel flows [12-15] and have found it very difficult to obtain statistically convergent results under experimental conditions of interest in MEMS [2-10]. Unlike hypersonic problems, microdevices often operate at low or moderate Mach numbers, which are characterized by a small ratio of macroscopic to thermal velocity. Because the statistical scatter inherent in DSMC decreases with the inverse square root of the sample size, an extremely large sample size is required to reduce it to a level that is small in comparison with the macroscopic velocity. This makes DSMC simulation of MEMS flows extremely time-consuming. Actually such a simulation is beyond the capabilities of current computers $[13,15]$.

An information preservation (IP) technique has been proposed to address this issue [16]. It assigns each simulated molecule in the DSMC method two velocities. One is the thermal velocity used to compute molecular motion following the same steps as the DSMC method. The other is called information velocity; it corresponds to the collective velocity of the enormous number of real molecules that the simulated molecule represents. Macroscopic velocity and shear stress are computed from the information velocity to remove the statistical scatter source inherent in the DSMC method due to the randomness of the thermal velocity. A preliminary study [16] showed that the IP technique was highly effective in reducing the statistical scatter.

In this paper, the IP technique will be systematically studied. First, the statistical fluctuation inherent in the DSMC method will be analyzed. Second, the IP technique will 
be described, and molecular collision diameters in accordance with the IP technique will be computed using experimental data on viscosity coefficients. Next, the IP method will be applied to benchmark problems. Finally, conclusions will be given.

\section{STATISTICAL SCATTER INHERENT IN THE DSMC METHOD}

As with most molecular-based numerical methods, the DSMC method suffers from statistical scatter. Scatter is generally regarded as the most serious practical and theoretical difficulty associated with this method. As pointed out by many researchers [12-15], such a difficulty becomes insurmountable for low-speed flows.

To clarify this fact, let us look at how the DSMC method computes macroscopic velocity $\mathbf{U}_{\ell}$ in a cell. Consider a uniform flow with velocity $\mathbf{u}_{k} ;$ then

$$
\mathbf{U}_{\ell}=\frac{1}{N} \sum_{k=1}^{N} \mathbf{c}_{k}=\mathbf{u}_{k}+\frac{1}{N} \sum_{k=1}^{N} \mathbf{c}_{t, k}
$$

where $N$ is the sample size of simulated molecules in the cell, and $\mathbf{c}_{k}$ is the molecular velocity. According to kinetic theory, $\mathbf{c}_{k}$ consists of two parts: the thermal part $\mathbf{c}_{t, k}$ and the macroscopic part $\mathbf{u}_{k}$. The expression $\mathbf{c}_{t, k}$ is random and obeys a Maxwellian distribution in an equilibrium gas. The DSMC method stores $\mathbf{c}_{k}$ in a computer and uses it to compute both the molecular trajectory and $\mathbf{U}_{\ell}$. Note that there is an explicit term $\frac{1}{N} \sum_{k=1}^{N} \mathbf{c}_{t, k}$ in Eq. (2) that makes $\mathbf{U}_{\ell}$ different from the exact value of $\mathbf{u}_{k}$. It is actually a source that results in the statistical fluctuation decreasing with the inverse square root of the sample size.

The order of this statistical scatter source is easily estimated through an actual DSMC simulation. Consider a stationary homogeneous argon gas at temperature of $273 \mathrm{~K}$. The values of macroscopic velocity given by the DSMC method in 100 independent runs, because of the existence of the scatter source, fluctuate around the exact value of zero. The $x$ components of the values against the sample sizes are partly given in Table I. For example, the absolute maximum $\sigma_{\max }$ at a sample size of $2 \times 10^{6}$ is $0.5301 \mathrm{~m} / \mathrm{s}$. This is unacceptable for many low-speed applications such as microchannel flows where the inflow velocity in experiments [4-10] was about $0.2 \mathrm{~m} / \mathrm{s}$. Further increasing the sample size is beyond the capabilities of current computers, particularly for multidimensional flows.

\section{INFORMATION PRESERVATION METHOD}

The IP technique assigns each simulated molecule in the DSMC method two velocities. One is the molecular velocity (i.e., $\mathbf{c}_{k}$ ), used to compute molecular motion following the same steps as the DSMC method. The other is called information velocity; this is the collective velocity of the enormous number of real molecules that the single simulated molecule represents, and therefore corresponds to $\mathbf{u}_{k}$. The term information velocity was invented to distinguish it from macroscopic velocity, which is understood as an average of molecular velocities over real molecules represented by many simulated molecules in the DSMC method. The IP technique employs the information velocity to compute the macroscopic velocity,

$$
\mathbf{U}_{\ell}=\frac{1}{N} \sum_{k=1}^{N} \mathbf{u}_{k}=\mathbf{u}_{k} \text {. }
$$




\section{TABLE I}

Statistical Fluctuation vs Sample Size in DSMC Simulation of a Stationary Homogeneous Argon Gas at 273 K

\begin{tabular}{cccccc}
\hline & \multicolumn{5}{c}{$u_{f}{ }^{a}$ in $\mathrm{m} / \mathrm{s}$ at different sample sizes } \\
\cline { 2 - 6 } No. of runs & $2 \times 10^{4}$ & $2 \times 10^{5}$ & $2 \times 10^{6}$ & $2 \times 10^{7}$ & $2 \times 10^{8}$ \\
\hline 1 & 2.3215 & -0.2243 & -0.1301 & 0.0904 & 0.0157 \\
2 & -0.4853 & -0.0825 & 0.1074 & -0.1038 & -0.0023 \\
3 & -2.5338 & -0.1724 & -0.1802 & -0.0397 & 0.0267 \\
4 & -2.0137 & 0.7793 & -0.1394 & 0.0138 & 0.0022 \\
5 & -0.8144 & 0.3451 & 0.1233 & 0.1202 & 0.0014 \\
$\cdot$ &. &. &. &. &. \\
$\cdot$ &. &. &. &. &. \\
98 & -1.2243 & -0.1722 & 0.0231 & -0.0722 & -0.0043 \\
99 & -1.5905 & -0.4683 & -0.0655 & -0.0207 & 0.0121 \\
100 & -5.0029 & 0.7412 & 0.0065 & -0.0665 & 0.0226 \\
$\sigma_{\max }$ & 6.2537 & 1.8344 & 0.5301 & 0.1546 & 0.0365 \\
\hline
\end{tabular}

${ }^{a} u_{f}$ is the $x$ component of the macroscopic velocity fluctuation caused by the statistical scatter, with the amplitude of $\sigma_{\max }$.

The statistical scatter source $\frac{1}{N} \sum_{k=1}^{N} \mathbf{c}_{t, k}$ in the DSMC formula (2) is removed in the IP formula (3). For a uniform flow, the macroscopic velocity calculated using (3) is the exact value of $\mathbf{u}_{k}$ at any sample size. Since the collective information propagates directly through molecular motion, a correct transport rate is expected over the entire Knudsen regime.

An implementation of the IP method can be summarized as follows:

(1) Assign simulated molecules an information velocity $\mathbf{u}_{k}$ and molecular velocity $\mathbf{c}_{k}$.

(2) Set the initial value of $\mathbf{u}_{k}$ to be the initial flow velocity.

(3) Move the molecules using $\mathbf{c}_{k}$ with the same algorithms and models as the DSMC method that have been described in detail in Ref. [11].

(4) In a time step $\Delta t, \mathbf{u}_{k}$ may be changed as a result of the following causes:

(4a) Impact with a wall. Set the reflected information velocity in accordance with the statistical behavior of an enormous number of real molecules. For example, it is equal to the wall velocity for a diffusely reflecting surface.

(4b) Entry into the computational domain from the boundaries. Set the information velocity in accordance with the boundary conditions.

(4c) Acceleration by external forces acting on a cell,

$$
\mathbf{a}=\frac{\mathbf{F}}{\rho \Delta V},
$$

where $\mathbf{F}$ is the sum of the external forces, and $\rho$ and $\Delta V$ are the density and volume of the cell, respectively. The acceleration will contribute a velocity increment $\mathbf{a} \cdot \Delta t$ to every simulated molecule within the cell.

(4d) Collision with other particles. A simple scheme satisfying momentum conservation is employed to distribute postcollision information velocities of two simulated particles,

$$
\mathbf{u}_{i, 1}^{\prime}=\mathbf{u}_{i, 2}^{\prime}=\frac{m_{1} \mathbf{u}_{i, 1}^{*}+m_{2} \mathbf{u}_{i, 2}^{*}}{m_{1}+m_{2}},
$$


where superscripts * and ' denote pre- and postcollision, respectively. An alternative scheme is to keep the information velocities unchanged during the collision process. Both schemes were tested in simulation of Couette, Poiseuille, and Rayleigh flows, and both gave identical results. This is easily understood because the scattering of the thermal velocities during the collision process is isotropic in the center of mass frame of reference according to the variable hard sphere (VHS) model $[11,17]$, which is used to describe the interaction between simulated particles. Such an isotropic scattering makes schemes that satisfy the momentum conservation the same in a statistical sense.

(5) Compute macroscopic quantities using the information quantities. The shear stress on a surface element with area $\Delta A_{w}$ during a sampling interval $t_{s}$ is equal to

$$
\tau_{w}=\frac{\sum_{k=1}^{N_{w}} m_{j}\left(u_{\tau, k}^{i n}-u_{\tau, k}^{r e}\right)}{t_{s} \Delta A_{w}},
$$

where $N_{w}$ is the number of molecules hitting the element during $t_{s}$, subscript $\tau$ denotes the tangential direction of the element, and superscripts in and re denote the values before and after striking the element, respectively. The macroscopic velocity in a cell is an arithmetic mean of all sampling information velocities during $t_{s}$ and is obtained using Eq. (3).

(6) For steady flows, repeat steps 3 and 4 until the flow reaches a steady state. Then repeat steps 3-5 to sample and obtain the macroscopic velocity distribution and shear stress. For unsteady flows, repeat steps 2-5 over the evolution period to obtain the macroscopic quantities period through ensemble average.

\section{MOLECULAR COLLISION DIAMETERS}

Molecular interaction is usually weakly attractive at a large distance and strongly repulsive at a short distance. An approximation is to take it into account only when the distance decreases to a finite cutoff value that is defined as molecular collision diameter. It is known that, to obtain accurate information from kinetic analysis and calculation, collision diameters must be chosen to give agreement between kinetic and experimental data for some basic quantity, such as viscosity coefficient [18]. Therefore, they are not universal but depend on molecular interaction models. For instance, the collision diameters given by the variable hard sphere (VHS) model [17] and by the variable soft sphere model [19], which are often employed in DSMC calculations, are different.

The IP technique computes postcollision information velocities according to Eq. (5), whereas the DSMC method distributes them along with the thermal velocities. This difference between the molecular interactions may make the collision diameters in accordance with the DSMC and IP methods slightly different. A procedure to determine the collision diameters in accordance with IP using experimental data on viscosity is given in Appendix A.

The hard sphere model assumes that the collision diameter of a molecule is a constant and the scattering from molecular collision is isotropic in the center of mass frame of reference. It is often employed in kinetic studies [11, 18, 20-23]. The procedure given in Appendix A has been applied to compute IP collision diameters $d_{H S}$ under this model. The values for five common gases ( $\mathrm{He}, \mathrm{Ar}, \mathrm{N}_{2}, \mathrm{O}_{2}$, and $\mathrm{CO}_{2}$ ) obtained using experimental data on the coefficients of viscosity at $273 \mathrm{~K}$ [18] are given in Table II. The corresponding shear stress distributions along the $y$ direction in the upper half channel of the Couette flow are given 
TABLE II

Collision Diameters in Accordance with the IP Method at a Reference Temperature of $273 \mathrm{~K}$

\begin{tabular}{lccccc}
\hline & $\mathrm{He}$ & $\mathrm{Ar}$ & $\mathrm{N}_{2}$ & $\mathrm{O}_{2}$ & $\mathrm{CO}_{2}$ \\
\hline$d_{H S} \times 10^{10}(\mathrm{~m})$ & 2.365 & 3.693 & 4.128 & 3.948 & 5.013 \\
$d_{r e f} \times 10^{10}(\mathrm{~m})$ & 2.463 & 4.283 & 4.312 & 4.295 & 5.620 \\
$\omega^{[18]}$ & 0.657 & 0.811 & 0.738 & 0.773 & 0.933 \\
\hline
\end{tabular}

in Table III. The resulting coefficients of viscosity $\mu_{\text {cal }} \times 10^{5}\left(\mathrm{~N} \mathrm{sm}^{-2}\right)$ are 1.876, 2.135, $1.660,1.919$, and 1.388 , respectively, and compare well with the measured data 1.865 , $2.117,1.656,1.919$, and 1.380 [18].

Coefficients of viscosity in the hard sphere model are proportional to the temperature to the power of 0.5 . For real gases, however, the power $\omega$ often significantly differs from 0.5. (The values of $\omega$ for many gases were given in Table 14 in [18], and those for He, $\mathrm{Ar}, \mathrm{N}_{2}, \mathrm{O}_{2}$, and $\mathrm{CO}_{2}$ are included in Table II.) To match this real-gas characteristic, the VHS model was introduced [17]. It assumes that the scattering from molecular collision is isotropic in the center of mass frame of reference, whereas the collision diameter is a function of the relative velocity $c_{r}$,

$$
d=d_{r e f}\left[\frac{\left(2 k T_{r e f} / m_{r} c_{r}^{2}\right)^{\omega-0.5}}{\Gamma(2.5-\omega)}\right]^{1 / 2}
$$

where $d_{\text {ref }}$ is the reference collision diameter at reference temperature $T_{\text {ref }}$ and $m_{r}$ is the reduced mass. Using the procedure given in Appendix A, $d_{r e f}$ in accordance with IP has

\section{TABLE III}

Shear Stress Distributions for Five Gases in the Upper Half Channel of the Couette Flow $\left(K n=0.01, u_{w}=1 \mathrm{~m} / \mathrm{s}, T=273 \mathrm{~K}\right)$ Given by IP Calculations with the Hard-Sphere Model

\begin{tabular}{|c|c|c|c|c|c|}
\hline \multirow[b]{2}{*}{$y / h$} & \multicolumn{5}{|c|}{ Shear stress $\left(\mathrm{N} / \mathrm{m}^{2}\right)$} \\
\hline & $\mathrm{He}$ & $\mathrm{Ar}$ & $\mathrm{N}_{2}$ & $\mathrm{O}_{2}$ & $\mathrm{CO}_{2}$ \\
\hline 0.0000 & 1.0412 & 3.2740 & 2.6896 & 2.9151 & 3.4353 \\
\hline 0.0333 & 1.0424 & 3.2723 & 2.6879 & 2.9133 & 3.4261 \\
\hline 0.0667 & 1.0436 & 3.2719 & 2.6880 & 2.9137 & 3.4187 \\
\hline 0.1000 & 1.0440 & 3.2715 & 2.6901 & 2.9107 & 3.4211 \\
\hline 0.1333 & 1.0423 & 3.2757 & 2.6908 & 2.9087 & 3.4266 \\
\hline 0.1667 & 1.0430 & 3.2659 & 2.6882 & 2.9061 & 3.4352 \\
\hline 0.2000 & 1.0482 & 3.2847 & 2.6827 & 2.8942 & 3.4289 \\
\hline 0.2333 & 1.0489 & 3.2641 & 2.6725 & 2.8976 & 3.4305 \\
\hline 0.2667 & 1.0482 & 3.2734 & 2.6973 & 2.9256 & 3.4254 \\
\hline 0.3000 & 1.0436 & 3.2918 & 2.7006 & 2.9209 & 3.4496 \\
\hline 0.3333 & 1.0485 & 3.2692 & 2.7301 & 2.9332 & 3.4601 \\
\hline 0.3667 & 1.0426 & 3.3026 & 2.7183 & 2.9286 & 3.4393 \\
\hline 0.4000 & 1.0477 & 3.2828 & 2.7262 & 2.9178 & 3.4444 \\
\hline 0.4333 & 1.0455 & 3.2933 & 2.7071 & 2.9201 & 3.4583 \\
\hline 0.4667 & 1.0447 & 3.2515 & 2.6936 & 2.9227 & 3.4461 \\
\hline
\end{tabular}


TABLE IV

Shear stress Distributions for Helium at Five Temperatures in the Upper Half Channel of the Couette Flow $\left(K n=0.01, u_{w}=1 \mathrm{~m} / \mathrm{s}\right)$ Given by IP Calculations with the VHS Model

\begin{tabular}{llllll}
\hline & \multicolumn{5}{c}{ Shear stress $\left(\mathrm{N} / \mathrm{m}^{2}\right)$} \\
\cline { 2 - 5 }$y / h$ & $89.7 \mathrm{~K}$ & $190.6 \mathrm{~K}$ & $256.7 \mathrm{~K}$ & $372.8 \mathrm{~K}$ & $456.7 \mathrm{~K}$ \\
\hline 0.0000 & 0.9144 & 0.9826 & 1.0297 & 1.0595 & 1.0923 \\
0.0333 & 0.9149 & 0.9855 & 1.0305 & 1.0585 & 1.0917 \\
0.0667 & 0.9154 & 0.9872 & 1.0312 & 1.0580 & 1.0926 \\
0.1000 & 0.9159 & 0.9898 & 1.0333 & 1.0572 & 1.0908 \\
0.1333 & 0.9160 & 0.9951 & 1.0359 & 1.0543 & 1.0888 \\
0.1667 & 0.9171 & 0.9968 & 1.0351 & 1.0557 & 1.0872 \\
0.2000 & 0.9161 & 0.9982 & 1.0348 & 1.0621 & 1.0866 \\
0.2333 & 0.9165 & 0.9961 & 1.0362 & 1.0576 & 1.0885 \\
0.2667 & 0.9182 & 0.9939 & 1.0336 & 1.0551 & 1.0870 \\
0.3000 & 0.9158 & 0.9957 & 1.0299 & 1.0563 & 1.0893 \\
0.3333 & 0.9134 & 0.9973 & 1.0291 & 1.0631 & 1.0902 \\
0.3667 & 0.9118 & 0.9881 & 1.0315 & 1.0606 & 1.0937 \\
0.4000 & 0.9116 & 0.9855 & 1.0336 & 1.0612 & 1.0967 \\
0.4333 & 0.9127 & 0.9940 & 1.0330 & 1.0656 & 1.0966 \\
0.4667 & 0.9101 & 0.9408 & 1.0295 & 1.0684 & 1.0976 \\
\hline
\end{tabular}

been computed. The resulting values of $d_{r e f}$ for the five common gases are given in Table II. The relation of shear stress and viscosity versus temperature may be studied with this model. The shear stress distributions along the $y$ direction in the upper half channel of the Couette flow for helium at five temperatures of 89.7, 190.6, 256.7, 372.8, and $456.7 \mathrm{~K}$ are given in Table IV. It is seen that the shear stress increases as the temperature increases. This is easily understood because larger thermal velocities at higher temperatures accelerate the frequency of momentum exchange between the gas and the plates. Figure 1 compares the relations of coefficient of viscosity of helium to temperatures given by the IP method, theory, and experiment [18], which agree quite well.

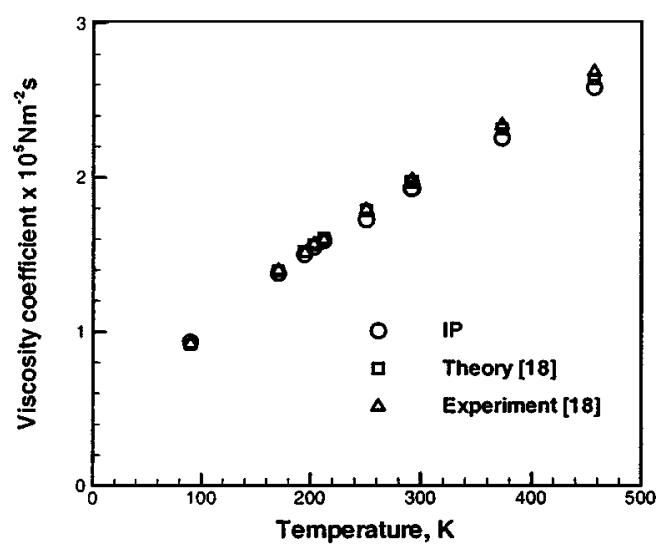

FIG. 1. Relation of viscosity coefficient of He versus temperature given by the IP method, theory, and experiment. 


\section{UNIDIRECTIONAL FLOWS}

Consider three typical unidirectional flows, namely, Couette, Poiseuille, and Rayleigh flows. They cover the most fundamental and important mechanisms that control a number of flows in MEMS applications. Because of the clear mechanisms, any drawbacks, if associated with a new method, will be easily found. Therefore, they have been widely used as benchmark problems to test and verify new analytical and numerical methods. Exact solutions are available at the continuum and free molecular limits for these flows, and there are analytical or numerical solutions of the linearized Boltzmann equation in the transition regime [20-23].

The linearized Boltzmann equation was analytically or numerically solved under the following assumptions [20-23]: (1) the gas molecules are hard spheres with uniform size and undergo complete elastic collisions between themselves; (2) the plate surfaces are diffusely reflecting; and (3) the disturbances are so small that the Boltzmann equation and boundary conditions may be linearized around an equilibrium state at rest. The IP simulated conditions are chosen to be consistent with these assumptions. The simulated gas is argon with temperature and pressure of $273 \mathrm{~K}$ and $0.01 \mathrm{~atm}$, respectively. The hard sphere model is employed to describe molecular interaction. The plate surfaces are assumed to be diffusely reflecting, with the same temperature as the gas. The plate velocity $u_{w}$ is $0.2 \mathrm{~m} / \mathrm{s}$ for the Couette flow and $1 \mathrm{~m} / \mathrm{s}$ for the Rayleigh flow. The pressure distribution along the $x$ direction for the Poiseuille flow is the same as that used in numerically solving the linearized Boltzmann equation [21],

$$
p=p_{o}(1+\alpha x / h)
$$

The values of $p_{o}$ and $\alpha$ are chosen to be 0.01 atm and $-2 \times 10^{-5}$, respectively, which result in a flow velocity of about $0.01 \mathrm{~m} / \mathrm{s}$. The characteristic velocities in the three flows are all very small in comparison with the thermal velocity of about $340 \mathrm{~m} / \mathrm{s}$. Such small flow velocities not only are of great interest in many MEMS applications, but also may strictly test the ability of the IP technique to reduce the statistical fluctuation in the conventional DSMC method.

\subsection{Couette Flow}

The Couette flow is a steady flow that is driven by the surface shear stresses of two infinite and parallel plates moving oppositely along their own planes (Fig. 2). The Knudsen number
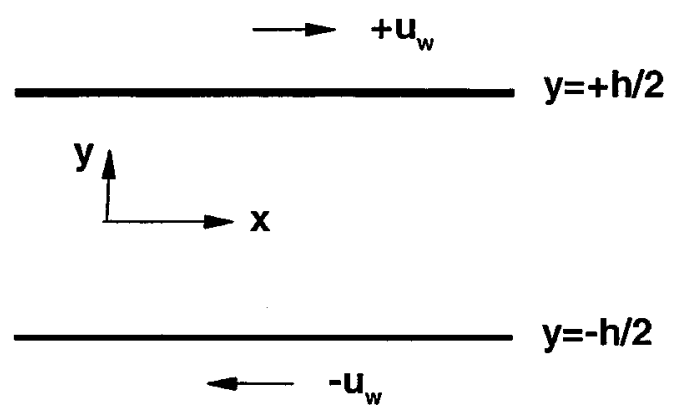

FIG. 2. Schematic diagram of the Couette flow. 
TABLE V

Computational Parameters for the Couette and Poiseuille flows ${ }^{a}$

\begin{tabular}{lrcccc}
\hline \multicolumn{1}{c}{$K n$} & 0.01 & $0.2 / \sqrt{\pi}$ & $2 / \sqrt{\pi}$ & $20 / \sqrt{\pi}$ & 100 \\
\hline$N_{m}$ & 9,000 & 900 & 900 & 900 & 900 \\
$N_{c}$ & 300 & 30 & 30 & 30 & 10 \\
$\Delta \ell / \lambda_{H S}$ & 0.33 & $2.95 \times 10^{-1}$ & $2.95 \times 10^{-2}$ & $2.95 \times 10^{-3}$ & 0.001 \\
\hline
\end{tabular}

${ }^{a}$ The time step is $0.3 \lambda_{H S} / v_{m}$, with $v_{m}=\sqrt{2 k T / m}$.

is defined as $K n=\lambda_{H S} / h$, where $h$ is the distance between the plates and $\lambda_{H S}$ is the mean free path, which is about $6.36 \times 10^{-6} \mathrm{~m}$ under the present conditions $(0.01 \mathrm{~atm}$ and $273 \mathrm{~K})$.

The IP simulation starts from a stationary uniform flow field. The computational parameters are given in Table $\mathrm{V}$, where $N_{m}$ is the total number of simulated molecules, $N_{c}$ is the number of cells, and $\Delta \ell$ is the cell size. When the flow evolves and reaches a steady state, the code starts to sample. The macroscopic velocity and surface shear stress are obtained according to step 5 in Section 3.

In the transition regime, three Knudsen numbers are considered: $0.2 / \sqrt{\pi}, 2 / \sqrt{\pi}$, and $20 / \sqrt{\pi}$. To resolve the flow fields well, 30 cells are employed in IP calculation for all the three cases. With a slip velocity model

$$
\left.u\right|_{y= \pm h / 2}= \pm\left. u_{w} \mp \frac{2-\sigma}{\sigma} \lambda_{H S} \frac{d u}{d y}\right|_{y= \pm h / 2},
$$

the velocity distribution across the channel given by the Navier-Stokes equation is

$$
\frac{u_{N S}}{u_{w}}=\frac{2}{1+2(2-\sigma) K n / \sigma} \frac{y}{h} .
$$

For the diffusely reflecting surfaces, the tangential momentum accommodation coefficient $\sigma$ is equal to 1 . Figure 3 compares the velocity profiles given by the IP method, the linearized

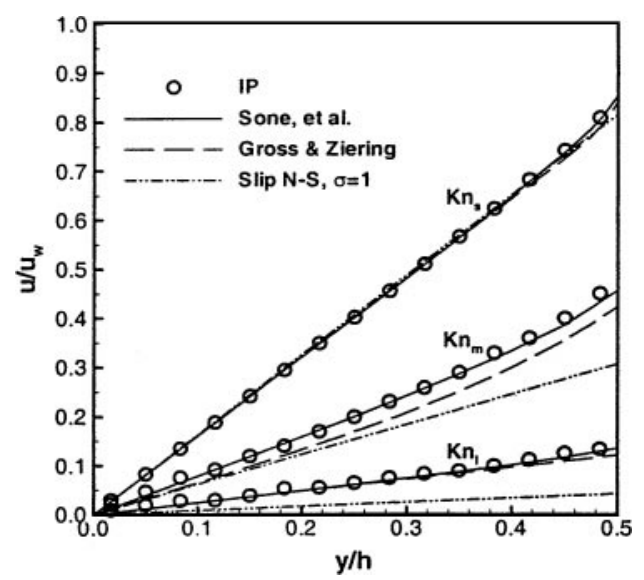

FIG. 3. Comparison of velocity profiles in the upper half channel of the Couette flow in the transition regime given by the IP method, linearized Boltzmann equation, and slip Navier-Stokes equations. $K n_{S}=$ $0.2 / \sqrt{\pi}, K n_{m}=2 / \sqrt{\pi}$, and $K n_{l}=20 / \sqrt{\pi}$. 


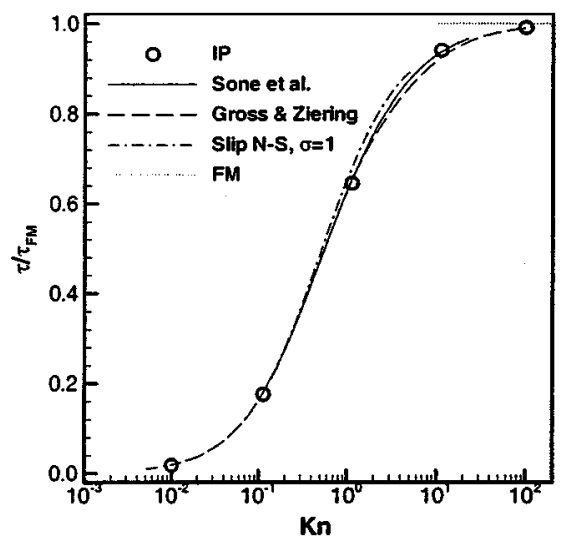

FIG. 4. Relation of drag versus Knudsen number for the Couette flow.

Boltzmann equation [20, 21], and Eq. (10). The velocity at the channel surface $(y / h=0.5)$ significantly decreases as $K n$ increases. The IP profiles are in excellent agreement with the numerical solutions of the linearized Boltzmann equation [21], which are more accurate than the four-moment solutions based on the second approximation [20]. The slip N-S profiles agree with the other three at $K n=0.2 / \sqrt{\pi}$, but deviate from them as $K n$ increases.

In the continuum regime $(K n=0.01)$, the IP and slip $\mathrm{N}-\mathrm{S}$ velocity profiles compare quite well; e.g., the gas velocities at the surfaces given by the IP method and model (9) are $\pm 0.9791 u_{w}$ and $\pm 0.9804 u_{w}$, respectively. In the free molecular regime $(K n=100)$, the gas velocity adjacent to the plate surfaces is only about $3 \%$ of $u_{w}$. Such a discontinuity is a typical phenomenon of very large Knudsen flows. During a time step of $0.3 \lambda_{H S} / v_{m}$, the average number of a simulated molecule colliding with the lower and upper plates is about 33. This means that the information velocity of a certain simulated molecule frequently leaps from $-u_{w}$ to $u_{w}$, or vice versa. The value at the sampling moment, i.e., at the end of the time step, is subject to the final collision that occurs at the lower or upper plate. The thermal velocity plays a role in such a process and therefore results in a statistical fluctuation. To reduce this fluctuation, $4 \times 10^{4}$ sampling time steps are employed in simulating the $K n=100$ case, much larger than 200 for the continuum case and 2000 for the transitional cases.

Figure 4 compares the relation of the surface shear stress versus the Knudsen number given by various methods. The normalization factor is the collisionless solution $\tau_{F M}=$ $\rho v_{m} u_{w} / \sqrt{\pi}$. The IP results agree quite with the exact solution in the free molecular regime, with the numerical Boltzmann solution [21] in the transition regime, and with the slip Navier-Stokes solution $(\sigma=1)$

$$
\frac{\tau_{N S}}{\tau_{F M}}=\frac{5 \pi K n}{8(1+2 K n)}
$$

in the continuum regime.

\subsection{Poiseuille Flow}

The plane Poiseuiile flow is a steady flow confined between two stationary infinite and parallel plates and is driven by a pressure gradient parallel to the plates (Fig. 5). The IP simulation starts from a uniform flow field. The acceleration that results from the pressure 


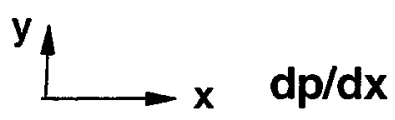

\section{$y=-h / 2$}

FIG. 5. Schematic diagram of the Poiseuille flow.

distribution (8) is $a=\alpha p_{o} / \rho h$. During each time step $\Delta t$, the $x$-components of both information and thermal velocities of simulated molecules obtain a velocity increment $a \Delta t$. Using the ideal gas equation of state, we have

$$
a \Delta t=0.5 \alpha v_{m} K n \Delta t^{*}
$$

where $\Delta t^{*}=v_{m} \Delta t / \lambda_{H S}$, and $K n$ has the same definition as in the Couette flow. On the other hand, the molecules are retarded when they collide with the stationary plates that are assumed to be diffusely reflecting: The information velocities become zero, while the molecular velocities are computed according to a Maxwellian distribution. The flow evolves and reaches a steady state when the acceleration and retardation are in balance.

Figure 6 shows the relation of the mass flux $Q_{M}$ with the Knudsen number given by the IP method, the linearized Boltzmann equation [22], experiment [24], and the $\mathrm{N}-\mathrm{S}$ equation with the slip boundary condition (1). $Q_{M}$ has been normalized by $\rho u^{*} h$, with $u^{*}=\alpha v_{m}$. The comparison of the IP mass flux with the numerical Boltzmann solution [22] and the experimental data [24] in the transition and near free molecular regimes is satisfactory. The slip Navier-Stokes mass flux $(\sigma=1)$,

$$
\frac{Q_{M}^{N S}}{\rho u^{*} h}=\frac{2\left(K n^{-1}+6\right)}{15 \sqrt{\pi}},
$$

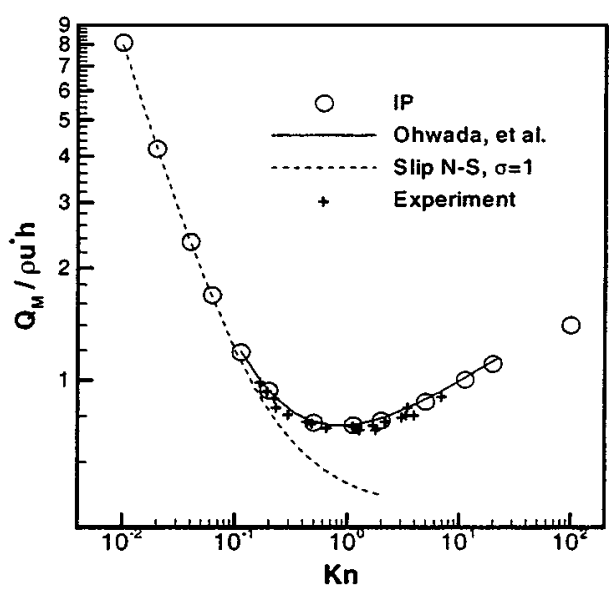

FIG. 6. Relation of the normalized mass flux versus Knudsen number for the Poiseuille flow. The 1st, 14th, and 18th experimental data from the small $K n$ side are for air, 2nd, 3rd, 7th, 10th, 13th, and 15th data for $\mathrm{CO}_{2}$, 4th, 8th, and 9th data for He, 5th, 11th, and 16th data for $\mathrm{H}_{2}$, and 6th, 12th, 17th, and 19th data for feron-12. 
agrees well with the IP results, as the Knudsen number is less than 0.05. There is a minimum mass flux at some intermediate Knudsen number. This minimum was first observed experimentally by Knudsen [25] and therefore is often referred to as the Knudsen minimum or the Knudsen paradox. The existence of such a minimum may be theoretically proven. The Navier-Stokes solution (13) shows that in the continuum regime the mass flux decreases as the Knudsen number increases, while the free molecular theory indicates that the mass flux through a duct with a finite length $L$ is proportional to the $\log$ arithm of $L / h$ [26]. Hence, the free molecular mass flux at a large enough $L / h$ will be higher than the continuum solution at a small but nonzero $K n$. Therefore, the minimum inevitably appears at some intermediate $K n$. The confirmation by the present IP calculation of the Knudsen minimum and its excellent agreement with the exact numerical solution of the linearized Boltzmann equation and experimental data near this minimum show clearly the fitness of the IP technique in predicting fine flow characteristics in the transition regime.

Figures $7 \mathrm{a}-7 \mathrm{c}$ show the velocity profiles obtained using the IP method, the linearized Boltzmann equation [22], and the slip Navier-Stokes equation at Knudsen numbers of $0.2 / \sqrt{\pi}, 2 / \sqrt{\pi}$, and $20 / \sqrt{\pi}$. The IP profiles compare well with the numerical Boltzmann
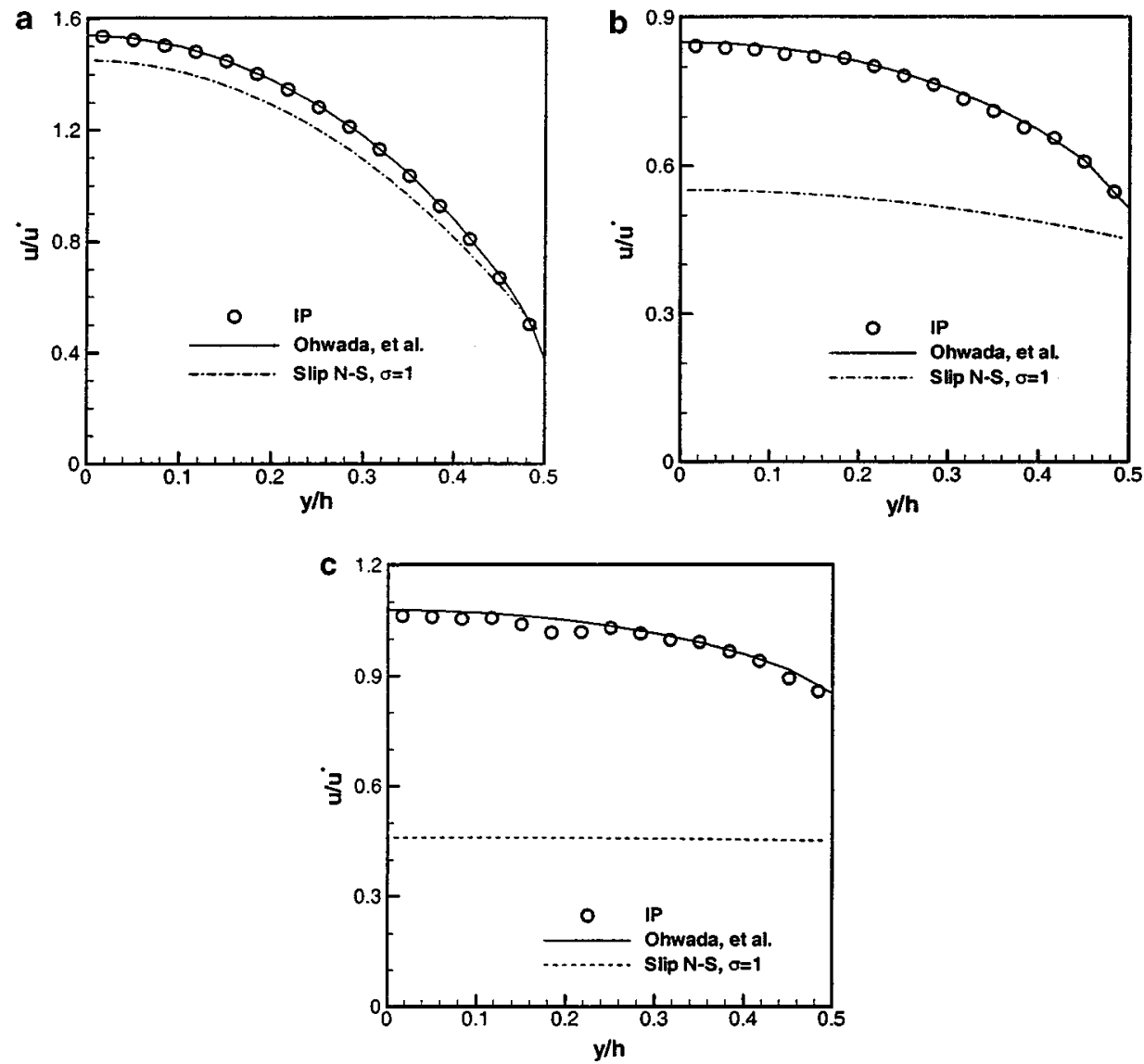

FIG. 7. Comparison of IP, linearized Boltzmann, and slip Navier-Stokes velocity profiles in the transition regime for the Poiseuille flow: (a) $K n_{S}=0.2 / \sqrt{\pi}$; (b) $K n_{m}=2 / \sqrt{\pi}$; (c) $K n_{l}=20 / \sqrt{\pi}$. 


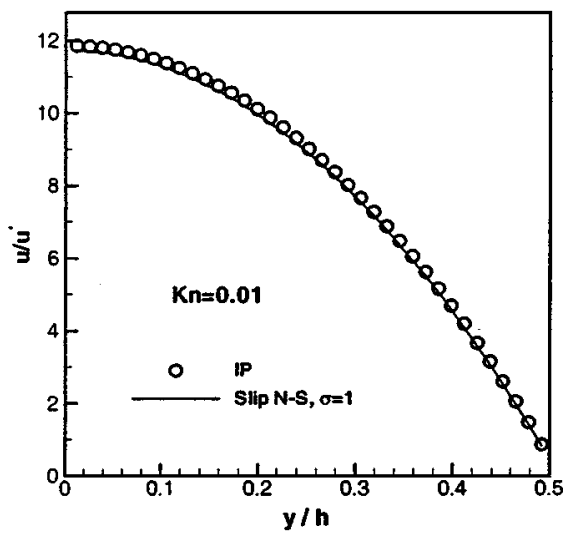

FIG. 8. Comparison of IP and slip N-S velocity profiles in the continuum regime $(K n=0.01)$ for the Poiseuille flow.

solution [22]. The slip N-S solution $(\sigma=1)$

$$
\frac{u_{N S}}{u^{*}}=\frac{1+4 K n-4 y^{2} / h^{2}}{5 \sqrt{\pi} K n}
$$

deviates from the two others as $K n$ increases.

Figure 8 compares the IP and slip N-S velocity profiles at $K n=0.01$, which agree with each other. Figure 9 shows the IP velocity profile at $K n=100$. There is a velocity discontinuity between the plate surfaces and the adjacent gas as expected. The number of sampling time steps is 500 for the Knudsen 0.01 case, 5000 for the transitional flows, and $2 \times 10^{5}$ for the Knudsen 100 case to reduce the statistical scatter caused by the frequent leaps of the simulated molecules between the lower and upper plates.

\subsection{Rayleigh Flow}

In the Rayleigh flow, the stationary plate acquires a velocity of $u_{w}$ in the $x$ direction at the initial time $(t=0)$. This impulsive motion of the plate induces an unsteady gas flow

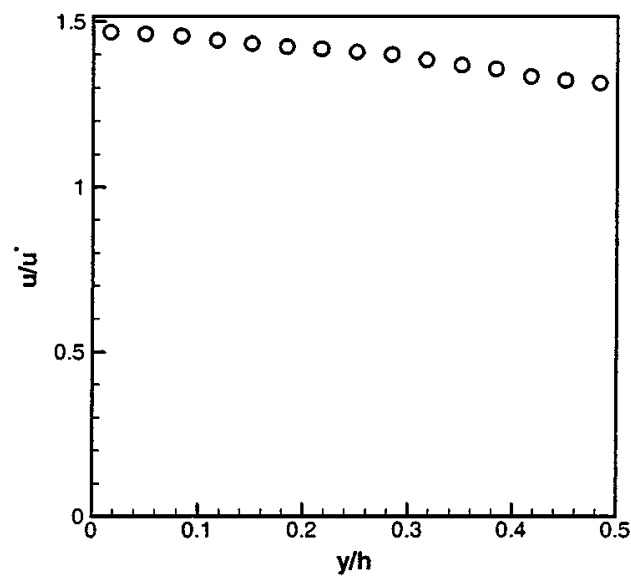

FIG. 9. IP velocity profile in the free molecular regime $(K n=100)$ for the Poiseuille flow. 


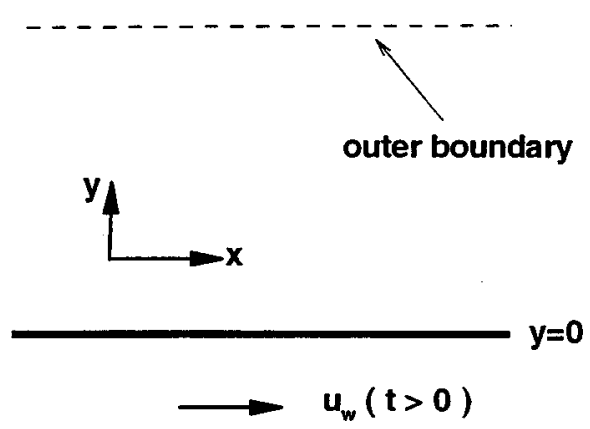

FIG. 10. Schematic diagram of the Rayleigh flow.

(Fig. 10). The ensemble average is employed in IP simulation of the unsteady process. The computational domain is between the plate surface and an outer boundary. The specularly reflecting condition is applied to the outer boundary. It is chosen far away from the plate to avoid possible backward disturbance to the gas motion near the plate. The computational parameters are given in Table VI, where $N_{s}$ is the sampling interval of time step.

For a time much less than the mean collision time $\tau_{o}\left(=\lambda_{H S} / v_{m}\right)$, few collisions take place. The situation is close to the free molecular flow that has the velocity distribution

$$
u_{F M}=0.5 u_{w} \operatorname{erfc}\left(y / v_{m} t\right)
$$

where erfc denotes the complementary error function.

For a time much longer than the mean collision time, so many collisions take place that the flow can be described by the Navier-Stokes equations. With a slip boundary condition

$$
\left.u\right|_{y=0}=u_{w}+\left.\lambda_{H S} \frac{d u}{d y}\right|_{y=0},
$$

the Navier-Stokes velocity solution may be written as [27]

$$
\frac{u_{N S}}{u_{w}}=\operatorname{erfc}\left(\frac{y^{*}}{2 \sqrt{a_{o} t^{*}}}\right)-\exp \left(y^{*}+a_{o} t^{*}\right) \operatorname{erfc}\left[\frac{y^{*}}{2 \sqrt{a_{o} t^{*}}}+\sqrt{a_{o} t^{*}}\right] \text {, }
$$

where $a_{o}=5 \sqrt{\pi} / 16, y^{*}=y / \lambda_{H S}$, and $t^{*}=t / \tau_{c}$.

The linearized Boltzmann equation has been solved using the four-moment method for two special cases [23]. The solution may be written as

$$
\frac{u_{4-m}^{S}}{u_{w}}=S\left(\phi_{1}\right)\left[0.139 e^{-b_{1} y^{*}}-6.10 \phi_{1} e^{-b_{1} y^{*}}\right]+S\left(\phi_{2}\right)\left[0.361 e^{-b_{2} y^{*}}+7.36 \phi_{2} e^{-b_{21} y^{*}}\right]
$$

TABLE VI

Computational Parameters for the Rayleigh Flow

\begin{tabular}{crrrccl}
\hline$t / \tau_{c}$ & \multicolumn{1}{c}{$N_{m}$} & $N_{c}$ & $N_{s}$ & $L_{o} / \lambda_{H S}$ & $\Delta \ell / \lambda_{H S}$ & $\Delta t / \tau_{c}$ \\
\hline 0.01 & 900 & 30 & 5 & 0.1 & $3.33 \times 10^{-3}$ & 0.002 \\
5 & 1,500 & 50 & 5 & 15 & 0.3 & 0.2 \\
100 & 24,000 & 800 & 5 & 240 & 0.3 & 0.2 \\
\hline
\end{tabular}




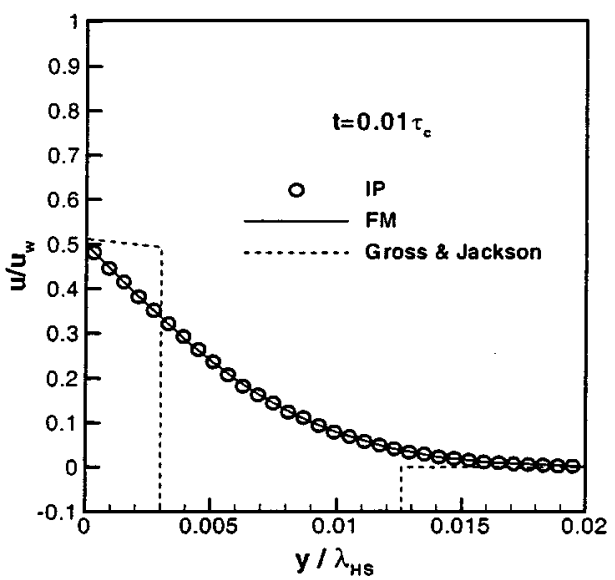

FIG. 11. Comparison of IP and theoretical velocity profiles at the free molecular limit for the Rayleigh flow.

for a short time $\left(t \ll \tau_{c}\right)$, and

$$
\begin{gathered}
\frac{u_{4-m}^{L}}{u_{w}}=\operatorname{erfc}\left(d_{0} y^{*} / 2 t^{*}\right)-1.24 \exp \left(d_{o}^{2} y^{*} / 4 t^{*}\right) / \\
\sqrt{\pi t^{*}}+0.292 \exp \left(-6.86 y^{*}\right) / \sqrt{\pi t^{*}}
\end{gathered}
$$

for a long time $\left(t \gg \tau_{c}\right)$, where $\phi_{1}=t^{*}-a_{1} y^{*}, \phi_{2}=t^{*}-a_{2} y^{*}, a_{1}=0.799, a_{2}=3.33$, $b_{1}=3.57, b_{2}=7.42, d_{o}=1.54$, and $S(z)$ is a step function that is equal to 0 and 1 for $z<0$ and $z>0$, respectively.

Figure 11 compares the velocity profiles at $t=0.01 \tau_{c}$ given by the IP method and Eqs. (15) and (18). The IP profile compares very well with the exact solution (15) of the collisionless theory. The four-moment profile is seen as discontinuous, and the part not shown slightly increases from $-5.9\left(y^{*}=0.0031\right)$ to $-5.7\left(y^{*}=0.0125\right)$. This discontinuity is not physically reasonable.

Figure 12 compares the velocity profiles at $t=100 \tau_{c}$ given by the IP method and Eqs. (17) and (19). The IP and slip N-S profiles are in excellent agreement, and the four-moment profile slightly differs from them. The difference between the four-moment solutions and exact solutions and the IP results may be caused by the poverty of the first approximation,

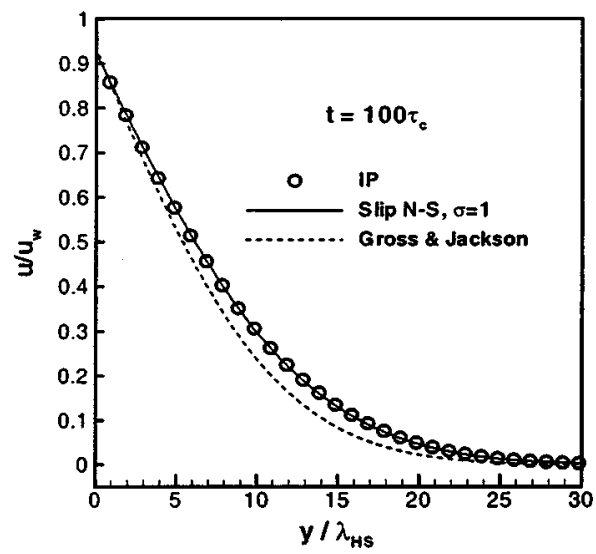

FIG. 12. Comparison of IP and theoretical velocity profiles at the continuum limit for the Rayleigh flow. 

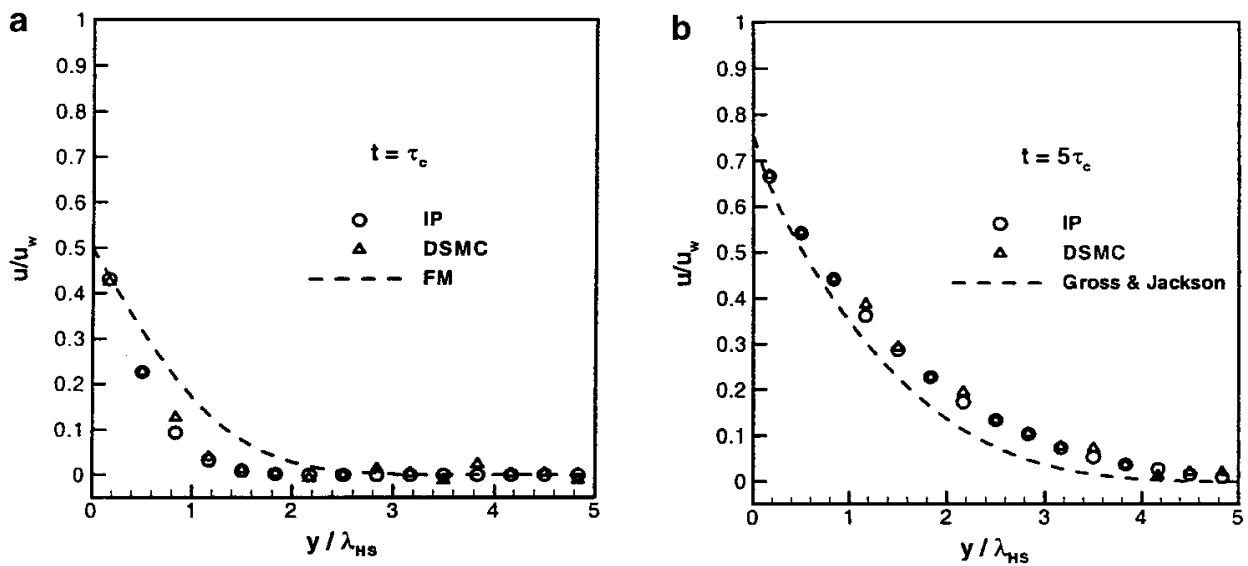

FIG. 13. Comparison of IP, DSMC, and theoretical velocity profiles in the transition regime for the Rayleigh problem. The DSMC sample size is $2 \times 10^{8}$, in comparison with the IP sample size of $6 \times 10^{3}$. (a) $t=\tau_{c}$; (b) $t=5 \tau_{c}$.

which was the only approximation employed in the analysis [23]. In fact, the first approximation solution for the Couette flow was found to deviate significantly from the second and third approximation solutions [20].

For a time comparable with the mean collision time, the DSMC method is employed to give a benchmark solution. Such a calculation, however, is very time-consuming. To reduce the DSMC statistical scatter to a level that is small in comparison with the characteristic velocity $u_{w}$ of $1 \mathrm{~m} / \mathrm{s}$, an enormous sample size of $2 \times 10^{8}$ is used. It takes about 180 CPU h on a DEC Alpha server 1000A, about $3 \times 10^{4}$ times as long as required by the IP method. Figures 13a and 13b compare the IP and DSMC velocity profiles at $t=\tau_{c}$ and $t=5 \tau_{c}$, respectively. The collisionless and four-moment, (15) and (19) are also shown as references. A satisfactory agreement is obtained between the IP and DSMC results, though some statistical fluctuation is still seen in the latter.

Figure 14 shows the relation of the normalized surface shear stress versus time given by the IP and other methods. The normalization factor is the free molecular solution

$$
\tau_{F M}=\frac{\rho v_{m} u_{w}}{2 \sqrt{\pi}} .
$$

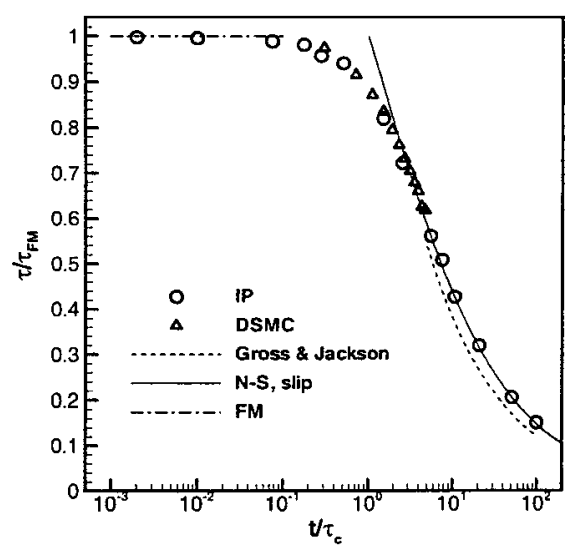

FIG. 14. Relation of drag versus time for the Rayleigh flow. 
The slip N-S solution obtained using Eqs. (16) and (17) is

$$
\frac{\tau_{N S}}{\tau_{F M}}=2 \sqrt{\pi} a_{o} \exp \left(a_{o} t^{*}\right) \operatorname{erfc}\left(\sqrt{a_{o} t^{*}}\right)
$$

The IP results agree quite well with the collisionless solution at $t \ll \tau_{c}$, with the DSMC results at $t \sim \tau_{c}$, and with the slip $\mathrm{N}-\mathrm{S}$ solution at $t>5 \tau_{c}$.

\section{CONCLUSIONS}

An information preservation technique was proposed to overcome the serious statistical fluctuation inherent in the DSMC method for low-speed rarefied gas flows. This technique was applied to benchmark problems, namely the Couette, Poiseuille, and Rayleigh flows, over the entire Knudsen regime. The characteristic velocities in these flows ranged from 0.01 to $1 \mathrm{~m} / \mathrm{s}$, which were much smaller than the thermal velocity of about $340 \mathrm{~m} / \mathrm{s}$. Meaningful results were obtained at a sample size of $10^{3}-10^{4}$, in comparison with a sample size of $10^{8}$ or more required for the DSMC method at such a range of flow velocity. This results in a tremendous gain in CPU time. A comparison of the velocity distributions, surface shear stresses, and mass fluxes given by the IP technique with exact solutions at the continuum and free molecular limits, and with numerical solutions of the linearized Boltzmann equation $[21,22]$, experimental data [24], and DSMC results in the transition regime, showed an excellent agreement.

\section{APPENDIX A}

\section{Procedure for Computing IP Collision Diameters}

Consider a plane Couette flow (Fig. 2). The plates move oppositely at a speed of $1 \mathrm{~m} / \mathrm{s}$. The IP simulated conditions are the same as those in experiments to measure viscosity, i.e., $101,32 \mathrm{~Pa}$ and $273 \mathrm{~K}$ [18]. The plate surfaces are assumed to be diffusely reflecting, with the same temperature as the gas. The distance between the plates is $100 \lambda_{H S}$, where $\lambda_{H S}$ is the molecular mean free path under the hard sphere model [18],

$$
\lambda_{H S}=\frac{16}{5 \sqrt{\pi}} \frac{\mu}{\rho v_{m}} .
$$

Here $\mu$ is the coefficient of viscosity, $v_{m}=\sqrt{2 k T / m}, m$ is the molecular mass, $k$ is the Boltzmann constant, and $\rho$ and $T$ are the gas density and temperature, respectively. The simulation employs 300 uniform cells, with 30 simulated molecules in each cell initially. The time step is $0.3 \lambda_{H S} / v_{m}$.

The simulation starts from an initial flow field with a linear velocity distribution. After 10,000 time steps, it reaches a steady state. Then the code starts to sample. The shear stress acting on each side of a cell is calculated as

$$
\tau_{x y}=\frac{\sum_{j=1}^{N^{-}} m_{j} u_{j}-\sum_{j=1}^{N^{+}} m_{j} u_{j}}{t_{s} \Delta A},
$$


where $N^{-}$and $N^{+}$denote the numbers of simulated molecules across the side from the negative and positive directions of $y$, respectively, $u$ is the $x$ component of information velocity, $t_{s}$ is the sampling time, and $\Delta A$ is the side area. The coefficient of viscosity results from

$$
\mu=\frac{\tau_{x y} \Delta y}{\Delta U},
$$

where $\Delta U=U_{o}-U_{a}, \Delta y=y_{o}-y_{a}, U_{o}$ and $U_{a}$ are the macroscopic velocities in the cell and its adjacent cell, and $y_{o}$ and $y_{a}$ are the $y$ coordinates of the cell centers. The coefficient of viscosity is then obtained by averaging over all cells except those in the Knudsen layer close to the surfaces.

For the hard sphere model, the molecular collision diameters are initially set to be

$$
d_{H S}=\left(\frac{5 m v_{m}}{16 \sqrt{2 \pi} \mu}\right)^{1 / 2} ;
$$

for the variable hard sphere (VHS) model [22], the reference collision diameters are initially set to be

$$
d_{r e f}=\left[\frac{5 \sqrt{m k T / \pi}}{4(5-2 \omega)(7-2 \omega) \mu}\right]^{1 / 2} .
$$

The calculated coefficient of viscosity decreases as the value of $d_{H S}$ or $d_{r e f}$ increases for the same type of gas. The acceptable value needs to satisfy the condition

$$
\left|\frac{\mu_{\text {cal }}}{\mu_{\text {exp }}}-1\right|<0.01,
$$

where $\mu_{\text {cal }}$ and $\mu_{\text {exp }}$ are the calculated and measured coefficients of viscosity, respectively.

\section{APPENDIX B}

\section{Computational Efficiency of the IP Method}

Since the IP technique is based on the DSMC method, we need to analyze the DSMC computational efficiency first. The total CPU time used in DSMC calculations may be expressed as

$$
T_{D S M C}=N_{\text {step }} \cdot \bar{T}_{\text {step }}
$$

where $N_{\text {step }}$ is the total number of time steps, $\bar{T}_{\text {step }}$ is the average CPU time spent each time step,

$$
\bar{T}_{\text {step }}=N_{\text {cell }} \cdot M_{\text {ave }} \cdot \bar{T}_{p}
$$

$N_{\text {cell }}$ is the number of cells, $M_{\text {ave }}$ is the average number of particles per cell, and $\bar{T}_{p}$ is the average CPU time spent per particle. $N_{\text {step }}$ has different expressions for steady and unsteady problems,

$$
N_{\text {step }}= \begin{cases}N_{\text {steady }}+N_{\text {sample }}, & \text { for steady } \\ N_{\text {loop }} \cdot N_{\text {single }}, & \text { for unsteady }\end{cases}
$$


where $N_{\text {steady }}$ is the number of time steps needed to evolve and reach a steady state from an initial state $N_{\text {sample }}$ is the number of sampling time steps, $N_{\text {loop }}$ is the number of independent runs for generating ensemble statistics, and $N_{\text {single }}$ is the number of time steps in a single run, which is the ratio of the evolution period $T_{E}$ to the time step $\Delta t . N_{\text {steady }}$ may be written as

$$
N_{\text {steady }}=\frac{\Delta P}{v \cdot \Delta t}
$$

where $\Delta P$ is the difference of a reference physical quantity $P$ at the initial and steady states and $v$ is the averaged relaxation rate of $P$ before reaching the steady state. Using the ratio of the required sample size $N_{\text {size }}$ to the average number of particles per cell $M_{\text {ave }}$ to estimate $N_{\text {sample }}$ and $N_{\text {loop }}$, we have

$$
T_{D S M C}= \begin{cases}\left(\frac{M_{a v e} \cdot \Delta P}{v \cdot \Delta t}+N_{\text {size }}\right) \cdot N_{\text {cell }} \cdot \bar{T}_{p}, & \text { for steady } \\ N_{\text {size }} \cdot N_{\text {cell }} \cdot \bar{T}_{p} \cdot \frac{T_{E}}{\Delta t}, & \text { for unsteady. }\end{cases}
$$

$\bar{T}_{p}$ consists of the following parts: that for selecting a collision partner and calculating the postcollision velocities, internal energies, etc., $\bar{T}_{\text {coll }}$; that for tracing its trajectory and indexing its cell number, $\bar{T}_{t-i}$; and that for sampling its velocity, etc., $\bar{T}_{\text {sample }}$. IP and DSMC have the same $\bar{T}_{t-i}$ for the algorithms to compute molecular trajectories, and cell indexes are identical to them. They also have the same $\bar{T}_{\text {sample }}$, for the number of operations to compute macroscopic physical quantities, such as flow velocity and shear stress from IP velocities, is the same as that from thermal velocities. The IP algorithm, resulting from Eq. (3), results in one more arithmetic mean operation in $\bar{T}_{\text {coll }}$ to compute the postcollision information velocities. Such an increase is small in comparison with the total number of operations to complete a molecular collision. Therefore, the values of $\bar{T}_{p}$ in the IP and DSMC methods are close.

The IP technique may greatly reduce the required sample size $N$ for low-speed flows in comparison with the DSMC method; e.g., $N_{\text {size }}$ is decreased $10^{4}$ for the Rayleigh flow described in Section 5. Therefore, the DSMC-based IP scheme, as stated by Eq. (B5), is quite helpful in improving the computational efficiency.

\section{ACKNOWLEDGMENTS}

This research was supported by Chinese Academy of Sciences Grant KJ 951-1-202 and National Natural Science Foundation of China Grants 19382009, 19772059, 59876043, and 19889209.

\section{REFERENCES}

1. H. Helvajian, Microengineering Aerospace Systems (Aerospace Press, 1999).

2. J. Pfahler, J. C. Harley, H. Bau, and J. N. Zemel, Gas and liquid flow in small channels, ASME-DSC 32, 49 (1991).

3. J. C. Harley, Y. Huang, H. Bau, and J. N. Zemel, Gas flow in micro-channels, J. Fluid Mech. 248, 257 (1995).

4. K. C. Pong, C. M. Ho, J. Q. Liu, and Y. C. Tai, Non-linear pressure distribution in uniform micro-channels, ASME-FED 197, 51 (1994). 
5. E. B. Arkilic, K. Breuer, and M. A. Schmidt, Gaseous slip flow in long microchannels, J. MicroElectroMechanical Syst. 6, 167 (1995).

6. J. C. Shih, C. M. Ho, J. Liu, and Y. C. Tai, Non-linear pressure distribution in uniform microchannels, ASME-AMD, 238 (1995).

7. J. C. Shih, C. M. Ho, J. Q. Liu, and Y. C. Tai, Monatomic and polyatomic gas flow through uniform microchannels, ASME-DSC 59, 197 (1996).

8. E. B. Arkilic, M. A. Schmidt, and K. S. Breuer, Measurement of the TMAC in silicon microchannels, in Rarefied Gas Dynamics, edited by C. Shen (Peking University Press, Beijing 1997), p. 983.

9. E. B. Arkilic, Measurement of the Mass Flow and Tangential Momentum Accommodation Coefficient in Silicon Microchannels, FDRL TR 97-1, Ph.D. thesis (MIT, 1997).

10. C. M. Ho and Y. C. Tai, Micro-electro-mechanical-systems (MEMS) and fluid flows, Annu. Rev. Fluid Mech. 30, 579 (1998).

11. G. A. Bird, Molecular Gas Dynamics and the Direct Simulation of Gas Flows (Clarendon, Oxford, 1994).

12. E. S. Piekos and K. S. Breuer, DSMC modeling of micromechanical devices, J. Fluids Eng. 118, 464 (1996).

13. C. K. Oh, E. S. Oran, and R. S. Sinkovits, Computations of high-speed, high Knudsen number microchannel flows, J. Thermophy. Heat Transfer 11, 497 (1997).

14. R. P. Nance, D. Hash, and H. A. Hassan, Role of boundary conditions in Monte Carlo simulation of MEMS devices, J. Thermophy. Heat Transfer 12, 447 (1997).

15. E. S. Oran, C. K. Oh, and Z. C. Cybyk, Direct-simulation Monte Carlo: Recent advances and applications, Annu. Rev. Fluid Mech. 30, 403 (1998).

16. J. Fan and C. Shen, Statistical simulation of low-speed unidirectional flows in transition regime, in Rarefied Gas Dynamics, edited by R. Brun, et al. (Cepadus-Editions, Toulouse, 1999), Vol. 2, p. 245.

17. G. A. Bird, Monte Carlo simulation in an engineering context, in Prog. Astronaut. Aeronaut. 74, 239 (1981).

18. S. Chapman, and T. G. Cowling, The Mathematical Theory of Non-uniform Gases, 3rd ed. (Cambridge Univ. Press, Cambridge, UK, 1970).

19. K. Koura and H. Matsumoto, Variable soft sphere molecular model for air species, Phys. Fluids A 4, 1083 (1992).

20. E. P. Gross and S. Ziering, Kinetic theory of linear shear flow, Phys. Fluids 1, 215 (1958).

21. Y. Sone, S. Takata, and T. Ohwada, Numerical analysis of the plane Couette flow of a rarefied gas on the basis of the linearized Boltzmann equation for hard-sphere molecules, Eur. J. Mech., B/Fluids 9, 273 (1990).

22. T. Ohwada, Y. Sone, and K. Aoki, Numerical analysis of the Poiseuille flow and thermal transpiration flows between two parallel plates on the basis of the linearized Boltzmann equation for hard-sphere molecules, Phys. Fluids 1, 2042 (1989).

23. E. P. Gross and S. Ziering, Kinetic theory of impulsive motion of an infinite plane, Phys. Fluids 1, 318 (1958).

24. W. Dong, Report UCRL-3353 (University of California, 1956, see also Ref. 19).

25. M. Knudsen, Ann. Phys., 28, 75 (1909).

26. Y. P. Pao, Courant Institute Report MF43 (New York University, 1964).

27. C. Shen, Lecture Notes on Rarefied Gas Dynamics (Chinese University of Science and Technology, 1962). 